\title{
Las arcillas de las formaciones geológicas de un área metropolitana, su uso en la industria cerámica e impacto en la economía regional
}

\author{
$\checkmark$ ANDERSON OSWALDO FLÓREZ-VARGAS ${ }^{1}$ \\ JORGE SÁNCHEZ-MOLINA² \\ DUVIN S. BLANCO-MENESES ${ }^{3}$
}

\section{Resumen}

El presente estudio realizó una síntesis de la situación en la industria cerámica del área metropolitana de Cúcuta con base al estado del arte proporcionado principalmente por organismos públicos; iniciando con una descripción de los depósitos de arcilla que son encontrados en las formaciones geológicas Leon y el grupo Guayabo, en el que el material exhibe características tecnológicas sobresalientes, producto de ello es la considerable participación de explotaciones mineras dentro del territorio, conformé a lo anterior se llevó a cabo un recuento de los títulos mineros en la base de datos de Catastro minero 2017, donde se encontró que Cúcuta es el municipio de mayor actividad extractiva, contando con abundantes reservas según se aprecian en las planchas de información geológica del SGC, contrario a Puerto Santander, municipio en el cual no existe actividad minera de arcilla ni manufacturas relacionadas a ella. La industria desarrollada a partir esta materia prima ha permitido un crecimiento económico en la región, situación que se refleja en la vinculación de 1.737 personas, 23 establecimientos creados y una producción bruta de más de 344 mil millones de pesos en el año 2015, siendo el principal grupo industrial de la zona metropolitana.

Palabras Claves: Arcilla, área metropolitana de Cúcuta, Formaciones, Minería, Industria, Economía.

1 Grupo de Investigación en Tecnología Cerámica de la Universidad Francisco de Paula Santander. San José de Cúcuta, Colombia.

2 Grupo de Investigación en Tecnología Cerámica, Centro de Investigación de Materiales Cerámicos (CIMAC), Universidad Francisco de Paula Santander. San José de Cúcuta, Colombia.

3 Empresa Cerámica Italia S.A. San José de Cúcuta, Colombia.

Autor de correspondencia: Flórez-Vargas, A.O. (Anderson

Oswaldo): Avenida Gran Colombia No. 12E-96 Barrio

Colsag, Of CIMAC. Teléfono: (+57) 3214024927

Correo electrónico: andersonflorez92@gmail.com
Historia del artículo:

Artículo recibido: 4-I-2018/ Aprobado: 17-V-2018

Disponible online: 3 de agosto 2018

Discusión abierta hasta abril de 2020 


\title{
Clays From Geological Formations of a Metropolitan Area, Its Use in the Ceramic Industry and Impact on the Regional Economy
}

\begin{abstract}
The present study made a synthesis of the ceramic industry situation in the metropolitan area of Cúcuta based on the state of the art provided mainly by public institutions; a description of the clay deposits that are found in the León and Guayabo geological formations is presented. Clays from these formations exhibits outstanding technological characteristics, therefore, considerable participation of mining operations are developed within this territory. A count of the mining titles was carried out in the 2017 cadastre database, where it was found that Cúcuta is the municipality with the greatest extractive activity, with abundant reserves as can be seen in the geological information maps of the SGC (Colombian Geological Service). On the other hand, Puerto Santander is the municipality in which there is no mining activity of clay or related manufactures. The industry developed from this raw material has allowed economic growth in the region, a situation that is reflected in 1,737 workers, 23 establishments created and a gross production of more than 344 billion of Colombian pesos in 2015, being the main industrial group of the metropolitan area.
\end{abstract}

Keywords: Clay, Metropolitan area of Cúcuta, Geological Formations, Mining, Industry, Economy.

\section{As argilas das formações geológicas de uma área metropolitana, seu uso na indústria cerâmica e impacto na economia regional}

\section{Resumo}

O presente estudo realizou uma síntese da situação na indústria cerâmica da área metropolitana da cidade de Cúcuta, baseado em dados proporcionados principalmente pelos organismos públicos; iniciando com uma descrição dos depósitos de argila que são encontrados nas formações geológicas Leon e o grupo Guayabo, em que o material exibe características tecnológicas notáveis, produto disso; é a participação considerável de explorações mineiras dentro do território. De acordo com acima exposto, uma contagem dos títulos de mineração foi feita tendo em conta a base de dados do Cadastro mineiro 2017, onde encontrou-se que Cúcuta é o município de maior atividade extrativa, possuindo abundantes reservas segundo se apreciam nas placas de informação geológica do SGC, contrário a Puerto Santander, município no qual não existe atividade mineira de argila nem manufaturas relacionadas com ela. A indústria desenvolvida a partir desta matéria prima tem permitido um crescimento económico na região, situação que se reflexa na vinculação de 1.737 pessoas, 23 estabelecimentos criados e uma produção bruta de mais de 344 mil milhões de pesos no ano de 2015, sendo o principal grupo industrial da área metropolitana.

Palavras-chave: Argila, Área Metropolitana de Cúcuta, Economia, Formações, Industria, Mineração. 


\section{Introducción}

El área metropolitana de Cúcuta se encuentra emplazada en medio de las distintas fuentes hídricas que componen la cuenca del Catatumbo, nombre que recibe en referencia a la principal arteria fluvial por donde confluye todas las aguas de la zona hacia el lago de Maracaibo (Venezuela), particularidad que ha distinguido a Cúcuta frente a las demás poblaciones fronterizas, desarrollando su capacidad comercial al punto de ser considerada una de las fronteras más activa de Latinoamérica, y uno de los puertos terrestres más importantes del país (Jiménez, 2008; Perdomo, Malaver, y Stalin, 2002; Urdanet y Maracaibo, 1988).

La cuenca del Catatumbo se compone de diversas microcuencas, entre las que se destacan la subcuenca del rio pamplonita y la del rio Zulia, cuya evolución geológica originó la aparición de formaciones sedimentarias litológicamente diversas, cabe señalar aquellas que se formaron en la edad del oligoceno y el mioceno llegando a extenderse hasta el plioceno, ya que en estos periodos se formaron capas de material arcilloso asociadas a lutitas, lodolitas, areniscas, entre otros, estos materiales son agrupados en la formación León durante la primera edad, y el grupo guayabo en los dos siguientes, según Van der Hammen (Como se cita en Instituto Colombiano de Geologia y Mineria, 1998). La calidad de las arcillas encontradas en estas formaciones ha provocado la aparición de empresas cerámicas dedicadas a la explotación de la materia prima para abastecer sus necesidades de consumo, dando como resultado productos con atributos deseados (Acuerdo 089 del 2011; Departamento Nacional de Planeacion, 2007).

Según Villamizar (2016), la explotación de arcillas es una de las actividades más activas del Departamento, llegando incluso a ser el segundo mineral de mayor producción, además de proporcionar los materiales para el desarrollo de la construcción, la cual es una de las actividades de más alto crecimiento (Sarabia-Guarin, Sánchez-Molina, y LeyvaDíaz, 2017). Es así como el gremio mantiene cifras significativas en despachos de productos terminado, en donde el mercado local representa un $25 \%$ de la oferta, la demanda nacional consume un $50 \%$ y el restante $25 \%$ se lleva a mercados extranjeros (Diaz, 2012), ofreciendo variedad de productos como tejas, bloques, ladrillos, tabletas, etc (Centro Regional de Estudios Económicos CREE Bucaramanga, 2005). A pesar de la ventaja competitiva que brinda el insumo, varios estudios (Banco de la República, 2005; Cely y Bolivar, 2015; Diaz, 2012) subrayan la necesidad de crear políticas que encaminen a fortalecer e incrementar el uso de herramientas tecnológicas en los procesos fabricación de productos cerámicos, especialmente el diseño de hornos (Sanchez y Diaz, 2011; J. Sánchez, Gelves, y Ramirez, 2012).

Debido a la gran importancia que representa esta temática para la región, el objetivo de esta investigación es caracterizar y relacionar los diferentes factores que se involucran en el sector cerámico en el área metropolitana de Cúcuta, describiendo el encadenamiento de los mismos, desde las fuentes naturales donde se encuentran las materias primas, las actividades de extracción, procesamiento y beneficio, y finalmente el impacto económico que genera a la comunidad.

\section{Metodología}

Para abordar la temática se partió de un reconocimiento cercano a la cadena de la arcilla en el departamento, se realizó contacto con los agentes que intervienen en el proceso como las unidades mineras, empresas cerámicas y organismos públicos. Para los primeros se tomó una muestra de 6 minas en explotación tomadas aleatoriamente, todas pertenecientes a un mismo grupo geológico, esparcidas dentro del área de estudio, a cada una de ellas se les realizó visita de campo donde se observó y tomo registro de sus operaciones, además se inspeccionó los resultados de calidad que proporcionan sus laboratorios de materias primas. Al segundo grupo, del total de las 67 empresas dedicadas a producir y comercializar artículos derivados de la arcilla (Cámara de comercio, 2012a), se seleccionaron 24 para 
entrevistar directivos y demás agentes de producción y así conocer más detalladamente sus técnicas de transformación y beneficio de los materiales, además de los costos que se generan, cabe resaltar que solo una éstas está dedicada a la producción de soportes esmaltados cerámicos, ya que es la única en su modalidad en el departamento, el restante desarrolla productos no esmaltados.

Los datos recolectados fueron agrupados y tabulados, seguidamente se analizaron para encontrar un explicación de los mismos, para ellos fue de gran ayuda las publicaciones de las instituciones públicas nacionales, departamentales y municipales que fueron el tercer agente consultados ya que suministraron información relevante del sector, se destaca el Servicio Geológico Colombiano, el Departamento Administrativo Nacional de Estadística, la Agencia Nacional Minera, las alcaldías municipales y la Cámara de Comercio de Cúcuta, los cuales brindaron estudios técnicos que permitieron referenciar las ideas plasmadas en este documento, adicionalmente se valió del estado del arte existente de investigaciones nacionales e internacionales, las cuales aportaron definiciones que actualmente se tiene sobre el tema. Todo esto con el objeto de contribuir al conocimiento de una de las actividades que produce gran dinamismo en Cúcuta y sus alrededores.

\section{Formaciones Geológicas en el área metropolitana de Cúcuta}

La geología presente en Cúcuta y su área metropolitana tiene su origen en los eventos geológicos que ocurrieron en la formación de la cuenca del lago de Maracaibo, considerado como el lago más grande Suramérica (Valera, 2016). Debido a su gran tamaño se divide en 33 subcuencas, destacándose la del Catatumbo(Ortega-lara, Lasso-alcalá, Lasso, Andrade de Pasquier, y Bogotá-gregory, 2012), ubicada hacia el suroccidente, la cual aporta el 60\% del agua dulce que llega al lago (Rivas et al., 2009).

La subcuenca del Catatumbo ocupa una superficie de $25600 \mathrm{~km}^{2}$ divididos entre Colombia y Vene- zuela (Ortega-lara et al., 2012). Para el Departamento Norte de Santander la subcuenca del Catatumbo es de suma importancia ya que sobre ella se extiende el 74,55\% de su territorio (Corporación Autonoma Regional de la Frontera Nororiental, 2011), y se concentra su población (Villamizar, 2016). Dado el gran tamaño que representa, ha sido necesario para el ente territorial, conocer la evolución e historia de la misma, que entre otras cosas permite distinguir los procesos de sedimentación, las edades de las rocas depositadas y los recursos minerales con los que cuenta (Vallejo, Ferrer, Ortuño, y Oteo, 2002). Esas necesidades conducen a investigaciones que son de gran utilidad para la divulgación de las riquezas provenientes del subsuelo, atrayendo el interés de empresas privadas y públicas. ejemplo de lo anterior, se aprecia en Bracho-Perez y Escobar-Navarro (2011) quienes describieron un posible origen de las reservas petroleras en la cuenca de Maracaibo a partir de la los cambios geoquímicos que ocurrieron de la conformación de las rocas, hacia el lado venezolano, considerado como el portador del $2 \%$ de las reservas mundiales de hidrocarburos (Barrero, Pardo, Vargas, y Martínez, 2007).

En Colombia, el Servicio Geológico Colombiano (SGC) es el encargado de regular el conocimiento geológico del suelo y subsuelo, actualmente brinda herramientas de información a la ciudadanía con la ayuda de portales interactivos, como ejemplo, la Figura 1 muestra el mapa geológico del área estudiada en el que se aprecia variedad de recursos pétreos. Adicional a esto, en el año 2016 el SGC logró avanzar en la generación de conocimientos por medio del uso de modernas tecnologías que le sirvió para completar la nueva versión geológica de la región (Servicio Geologico Colombiano, 2017a), la información está integrada por 6 cuadrantes que dan un mayor detalle respecto al mapa general de la Figura 1, denominados planchas No. 77, 78, 87, 88, 97 y 98, allí se muestra parte de la litología que conforma la columna estratigráfica de la cuenca del Catatumbo (Agencia Nacional de Hidrocarburos, 2010). En dicha columna se resaltan las formaciones León 
y Guayabo señaladas de poseer minerales de arcilla con atributos deseables para la industria del gres (Gelves, Peña y Sánchez, 2009), considerados la materia prima principal del proceso de fabricación cerámica (Campos, et al., 2006), especialmente el grupo Guayabo, ya que en él se encuentran arcillas de buenas características y en grandes cantidades (Freddy y Diaz, 2011; SIMEC, 2012).

Figura 1. Ubicación del área de estudio (comprendido por la zona encerrada por el trazo color rojo) y mapa geológico tomado de Servicio Geológico Colombiano (2017b) Leyenda: 1. Rocas calizas intercaladas con margas, lodolitas calcáreas y arenosas. 2. Intercalaciones de arenitas localmente conglomeriticas, lodolitas y arcillolitas. Ocasionalmente delgadas capas de carbón. 3. Arenitas de grano fino a conglomeriticas, interestratificadas con arcillolitas y limolitas, ocasionalmente algunos lentes de hierre y carbón. 4. Depósitos aluviales y llanuras aluviales. 5. Terrazas aluviales. 6. Abanicos aluviales y depósitos coluviales. 7. Shales, calizas, arenitas, cherts y fosforitas.

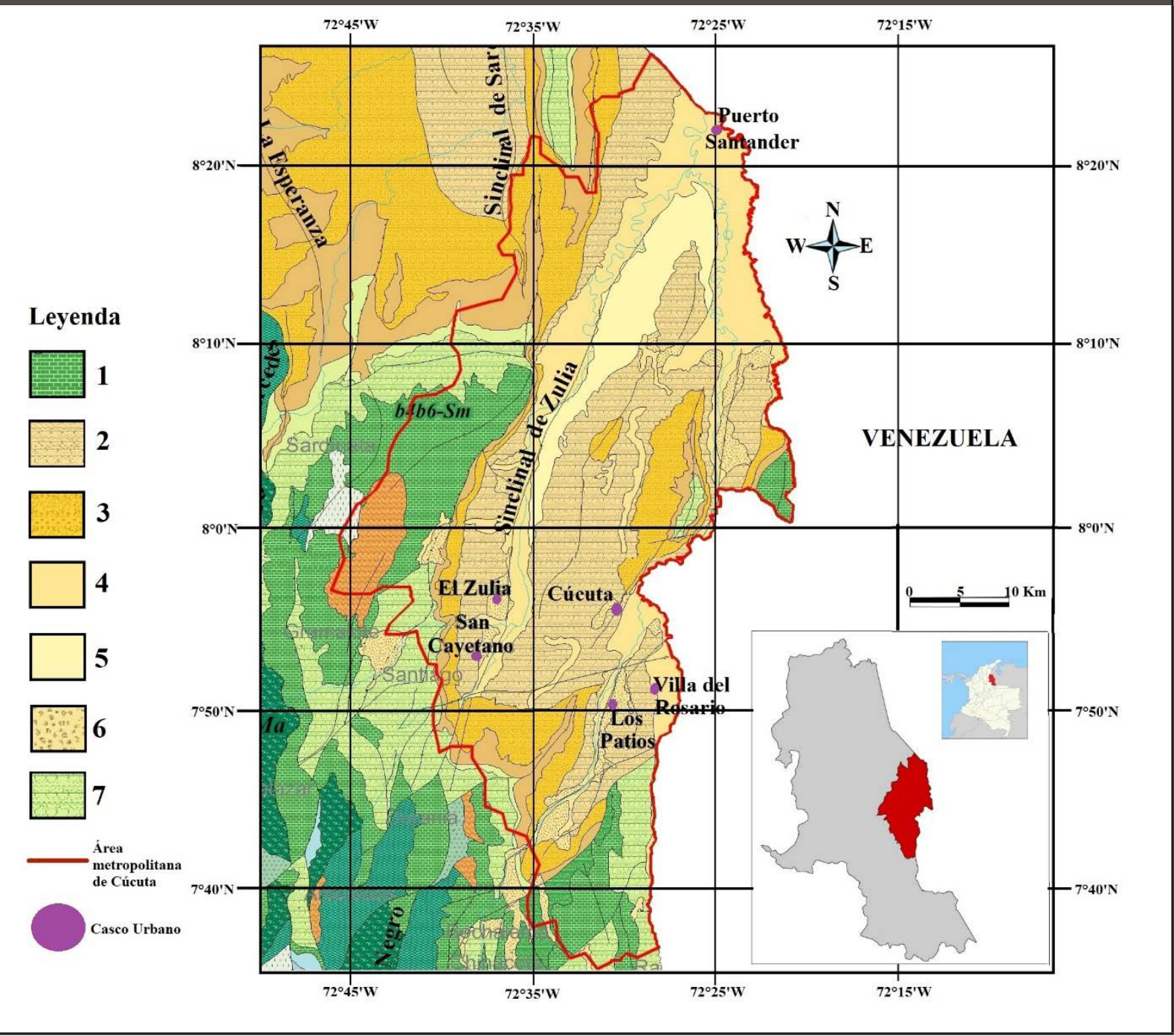

De acuerdo al Consorcio GSG (2015), el cuadrante 77 también llamado Campo Dos, ubicado hacia el noroeste, evidencia la ausencia de los grupos geológicos de interés en el área de estudio, contrario a ello en la plancha 78 que está al noreste, deja ver a las formaciones León y Guayabo inmersas en el área rural norte de Cúcuta, el primero forma el 7\% de la superficie de la plancha y está compuesto principalmente de lutitas color gris a verde, con intercalaciones de areniscas de color naranja y lodolitas, 
mientras el segundo resta un 14\% del cuadrante, asociado a dos gruesas capas de areniscas separadas por un estrato de arcillas moteadas de diversos colores, lo anterior concuerda con lo expuesto Figura 1, en donde también se observa rocas de tipo cuaternario como depósitos coluviales o aluviones, específicamente el municipio de Puerto Santander. Hacia sector centro oeste de la Figura 1, el cuadrante 87 revela la información geológica de los municipios del Zulia y San Cayetano, allí se aprecia la formación León únicamente en el sector occidental de El Zulia, constituido por shales de color gris a verdes, especialmente arcillosos en la base y el techo, mientras, el grupo Guayabo se presenta en una gran área al este del municipio, y hacia la parte norte de San Cayetano, su litología está compuesta de arenas lodosas, lodolitas arenosas, lodolitas arcillosas y arcillolitas vari coloreadas (Ochoa et al., 2016).

La Plancha 88, también llamada Cúcuta, muestra la formación León inmersa solamente en esa municipalidad, su composición es mayoritariamente lutitas grises a verdes, con cintas de arena y más común limolitas; en tanto que el grupo guayabo se describe como un gran bloque litológicamente diverso, siendo representativo las arcillolitas abigarradas, los estratos de areniscas y material de conglomerado, expuesto en gran parte en la zona oeste de Cúcuta y en sus proximidades con San Cayetano (Oviedo, Rios, García, y Ochoa, 2016). Finalmente, el sur del área metropolitana se representa en las planchas 97 y 98 , donde se evidencia la formación león aflorando en gran parte de Cúcuta y en menor proporción sobre los municipios de San Cayetano, el centro-norte de Los Patios y muy escasamente sobre Villa del Rosario, las rocas que componen esa formación son lodolitas grises a verdosas con algunas intercalaciones de areniscas. De manera opuesta, el grupo Guayabo abarca una gran extensión en los municipios de Villa del Rosario y los Patios, sin mayor presencia sobre el sur de Cúcuta y San Cayetano, las rocas de este grupo son descritas como areniscas blancas con intercalaciones de lodolitas grises (Instituto Colombiano de Geología y Minería, 2011).
La anterior descripción de las formaciones León y Guayabo muestra claridad de su constitución, la cual comprende variedad de rocas, con predominación de arenas, lodolitas, lutitas y arcillolitas, estas últimas regularmente son portadoras de minerales de arcilla. Debido a lo anterior y a la poca continuidad de los espesores en las unidades geológicas, especialmente del grupo guayabo, que en promedio se mantiene en $800 \mathrm{~m}$, aunque en algunos sectores de Cúcuta ha llegado a medir hasta2640 metros(Consorcio de estructuración Vial, 2014), sumado a la variación de las aptitudes de sus arcillas ha llevado a que la minería local en sus proyectos clasifique un conjunto de estratos de arcilla como bloques geológicos de interés económico si estos cumplen con estándares de calidad requeridos por los procesos industriales a los que vayan a ser sometidos, haciendo necesario en algunas ocasiones la extracción por separado de algunos mantos para hacer viable la explotación del macizo rocoso (Saavedra, 2016), de acuerdo de ello, la región no tiene definido un tipo de manto especifico como potencial de producción, ya que esto varia con los estratos acompañantes a la arcilla.

\section{La minería de la arcilla en el área metropolitana de Cúcuta}

Dada la ubicación geográfica de Cúcuta y sus poblaciones aledañas, la región cuenta con un acceso rápido a reservas considerables de material arcilloso y demás materias primas de excelentes características para la industria (Rozo Rincón, Sánchez Molina, y Gelves Díaz, 2015), permitiendo un aprovechamiento de las mismas a través de técnicas de explotación y demás procesos que le resultan competitivo (Cámara de comercio de Cúcuta, 2012). Para conseguir beneficios de estos materiales, todo interesado debe celebrar un contrato con el Estado, en donde por cuenta y riesgo del solicitante se desarrollan las etapas del proceso minero, que exige el pago de una contraprestación económica a la nación por ser la propietaria de los recursos naturales (Ley $685,2001)$. 
De acuerdo con la Agencia Nacional Minera (2017), Catastro Minero Colombiano (2017) y el Sistema de Información Minero Colombiano (2017), la actividad minera de la arcilla en el área metropolitana de Cúcuta cuenta con 138 títulos a mayo del 2017, distribuidos en 12.253,83 ha, en donde se destaca el municipio de Cúcuta, el cual cuenta con aproximadamente 4.635,18 ha del total metropolitano, siendo el de mayor participación en área concesible $(37,83 \%)$, seguido de Los Patios con $23,03 \%$, El Zulia 22,42\%, San Cayetano 10,50\%, y finalmente Villa del Rosario con 6,23\%. Se observa según las fuentes oficiales que en el municipio de Puerto Santander no hay presencia de títulos de arcillas, ni de otro mineral, en el periodo de estudio. La Tabla 1 muestra la información anteriormente expuesta; debe aclararse que el total de contratos de concesión dentro del área metropolitana son 138, a pesar de que la suma de los parciales de la columna número de Títulos relacionados dé como resultado un número mayor, esto se debe a que algunos títulos poseen participación en más de un municipio

TABLA 1. COMPOSICIÓN DE TÍTULOS MINEROS EN EL ÁREA METROPOLITANA DE CÚCUTA.

FUENTE: ELABORACIÓN PROPIA CON BASE A LA INFORMACIÓN SUMINISTRADA EN SITIO OFICIAL DE CATASTRO MINERO MAYO DEL 2017.

\begin{tabular}{c|c|c|c} 
Municipio & $\begin{array}{c}\text { Número } \\
\text { de Títulos } \\
\text { relacionados }\end{array}$ & $\begin{array}{c}\text { Área } \\
\text { otorgada } \\
\text { (ha) }\end{array}$ & $\begin{array}{c}\text { Participación } \\
\text { (\%) }\end{array}$ \\
\hline Cúcuta & 49 & 4635,18 & 37,83 \\
\hline Los Patios & 33 & 2821,70 & 23,02 \\
\hline El Zulia & 38 & 2746,97 & 22,42 \\
\hline San Cayetano & 10 & 1286,17 & 10,50 \\
\hline $\begin{array}{c}\text { Villa del } \\
\text { Rosario }\end{array}$ & 28 & 763,81 & 6,23 \\
\hline $\begin{array}{c}\text { Puerto } \\
\text { Santander }\end{array}$ & 0 & 0 & 0 \\
\hline $\begin{array}{c}\text { Total Área } \\
\text { Metropolitana }\end{array}$ & 138 & 12253,83 & 100 \\
\hline
\end{tabular}

Nota: Algunos títulos circundantes a Cúcuta y su AM, fueron excluidos por tener un porcentaje de participación muy insignificante en la misma.
Sánchez (2017) toma en cuenta el área total concedida por el estado para la explotación de arcilla descrita en la Tabla 1 y lo compara respecto a la superficie ocupada por el área metropolitana de Cúcuta, encontrando que el 6,25\% del territorio es utilizado para esa actividad, además realiza algunas estimaciones de las reservas de arcilla en la región, en donde a un ritmo de 53300 toneladas al mes de productos terminados, la región puede contar con más de 919 años del recurso mineral.

Además de los títulos vigentes a la fecha, las fuentes revelan la demanda de áreas para la extracción de arcilla en un total de 25 solicitudes, llama la atención que un $20 \%$ de las mismas son requeridas por explotadores vigentes que no poseen contrato de concesión, siendo necesario demostrar una continuación de trabajos mineros desde antes de agosto del 2001 (Decreto 2390, 2002).

\section{Propiedades físico-cerámicas de las}

\section{Arcillas frente a los procesos industriales}

El conocimiento de las propiedades tecnológicas de las arcillas, las cuales dependen en gran medida del tipo y composición de minerales, ayudan a comprender la tecnología de producción cerámica y la optimización de los ciclos de cocción (Milošević et al, 2017). Por lo tanto, puede esperarse que arcillas de distinta naturaleza presenten diferencias en sus parámetros cerámicos.

En el área metropolitana de Cúcuta la actividad minera se concentra en las arcillas presentes en el grupo geológico Guayabo, las cuales poseen características que resultan atractivas para la industria. Sin embargo, se ha notado variaciones en los parámetros cerámicos aun dentro de las arcillas de la misma formación, por lo que, las materias primas que ingresan a las plantas de beneficio con clasificadas según sus características y apiladas en distintos lotes, en donde el personal técnico y encargado de la elaboración de la pasta cerámica formulan porcentajes de participación a éstos, con el fin de dosificar la composición de la masa para la realización de una determinada pieza. 
El presente estudio analizó los resultados del comportamiento físico-cerámico de las arcillas presentes en seis unidades mineras ubicadas dentro de la región, pertenecientes al grupo Guayabo, se tomaron en cuenta los parámetros de porcentaje en contracción en cocido (\%CC), porcentaje de absorción de agua (\%AA), perdidas por fuego (\%PPF) y porcentaje de arena $(\% A)$, ya que son las variables de mayor control en las empresas.

El porcentaje de contracción en cocido indica la reducción en tamaño dimensional que va sufrir la pieza cerámica después la cocción.

El porcentaje de absorción de agua revela el grado de porosidad que el material puede presentar al retener en sus poros agua (Sacmi Iberica S.A., 2001) asimismo, es posible inferir la resistencia a la flexión que puede sufrir el producto bajo determinado intervalo de cargas, comportándose de manera inversamente proporcional (Alvarez Rozo y Sánchez Molina, 2014).

Finalmente, el porcentaje de pérdidas por fuego o perdidas por calcinación, consta de la eliminación de impurezas a través de la cocción de la pasta, en su mayoría material orgánico que se encuentra ligado a la arcilla, por lo que existe una disminución en el volumen y peso de la misma una vez haya salido del horno.

Los proyectos mineros considerados para el análisis se hallan esparcidos en el área de estudio, la mina A se ubica en el municipio de San Cayetano, la mina B en el Zulia, las minas C y D, ambas emplazadas sobre Cúcuta, los restantes dos títulos se ubican al sur, la mina E en Los Patios y la mina F sobre Villa del Rosario.

La Tabla 2 correlaciona los parámetros evaluados en cada uno de las minas analizadas, las pruebas se hicieron sobre probetas tabulares realizadas con la técnica de prensado, bajo un ambiente de cocción a $1140^{\circ} \mathrm{C}$ en un tiempo promedio de 35 minutos.

Según el grado de absorción de agua (ver Tabla 2) y dado que el método de fabricación de las probetas analizadas fue el mismo, es posible clasificar el material de las seis minas en dos grupos, donde las arcillas de las minas A, B, D y F se ubican en el grado de absorción medio-alto y las dos restantes son consideradas de nivel alto, ya que sus \%AA es mayor a 10 (Generalitat Valenciana e Instituto Valenciano de la Edificación, 2011). En consecuencia, de no realizarse mezclas que busquen homogeneizar el material en todos los títulos relacionados, los procesos de fabricación la baldosa cerámica en cada uno de ellos deben sujetarse a la norma establecida de acuerdo al tipo de grupo referido, obedeciendo criterios en calidad superficial, dimensiones, propiedades físicas y químicas distintos, para el caso colombiano rige la norma NTC 919 (Instituto Colombiano de Normas Tecnicas y Certificación, 2015).

TABLA 2. VALORES DE LOS PRINCIPALES PARÁMETROS FÍSICO-CERÁMICOS DE LAS ARCILLAS ENCONTRADAS EN LAS MINAS DESCRITAS.

FUENTE. ELABORACIÓN PROPIA CON BASE A LA INFORMACIÓN SUMINISTRADA POR LA EMPRESA TITULAR DE LAS MINAS.

\begin{tabular}{c|c|c|c|c|c|c|}
\hline Parámetro Físico Cerámico & $\begin{array}{c}\text { Mina A } \\
\text { San } \\
\text { Cayetano } \\
\text { Contracción en Cocido (\%CC) }\end{array}$ & $\begin{array}{c}\text { Mina B } \\
\text { El Zulia }\end{array}$ & $\begin{array}{c}\text { Mina C } \\
\text { Cúcuta }\end{array}$ & $\begin{array}{c}\text { Mina D } \\
\text { Cúcuta }\end{array}$ & $\begin{array}{c}\text { Mina E } \\
\text { Los Patios }\end{array}$ & $\begin{array}{c}\text { Mina F } \\
\text { Villa de } \\
\text { Rosario }\end{array}$ \\
\hline Absorción de Agua (\% AA) & 7,49 & 8,42 & 10,63 & 6,33 & 10,09 & 9,14 \\
\hline Perdidas por Fuego (\%PPF) & 5,62 & 5,64 & 4,86 & 5,99 & 5,30 & 5,66 \\
\hline \% Arena & 13,6 & 11,35 & 17,9 & 8,3 & 10,3 & 11,17 \\
\hline
\end{tabular}


La contracción de cocido tiene una relación inversamente proporcional con la cantidad de materiales desgrasantes que componen la arcilla, en donde a mayor contenido de éstos últimos, la contracción en el material cerámico es menor (Gelves, Monroy, Sanchez y Ramirez, 2013). Esta situación se presenta con los datos descritos, donde se aprecia que la mina $\mathrm{C}$ posee el valor más alto en el porcentaje de arena y a su vez presenta la menor contracción en cocido respecto a las demás, inverso a lo que se observa en la mina $\mathrm{D}$, donde el porcentaje de arena es menor al de todas unidades y consigue la mayor contracción en la cocción.

Las pérdidas por fuego fue el parámetro que mostró menor variación, teniendo un promedio de $5,51 \%$ y una dispersión de $\pm 0,57 \%$; a pesar de que no existe un valor específico para clasificar una arcilla como buena o mala de acuerdo a este factor, ya que también influyen las condiciones de fabricación de los materiales y el tipo cocción (siendo más sensibles aquellos de mono cocción) (Sánchez, García, Sanz, y Ochandio, 1990), varios autores señalan un límite de $0,3 \%$ en el carbono presente en la materia orgánica, en el que es de esperarse que el \%PPF sea proporcional a la cantidad de carbono orgánico, sin embargo no se ha encontrado una ecuación que modele dicha proporción ya que ésta puede variar acorde a los ambientes de formación de las arcillas, no obstante, Lores, Font, De la Torre, y Bastida (1997), en un estudio de la materia orgánica en 10 tipologías de arcillas, se evidenció que un aumento partir de 7,50 \% en pérdidas de calcinación el valor del contenido en carbono superaba el 0,3\%. De esperarse un comportamiento similar en las arcillas de las minas analizadas, los seis tipos de material estarían en condiciones favorables para la fabricación de baldosas, sin esperar a presentar defectos como el de núcleo negro, que es un signo desfavorable en la calidad del producto, aunque para obtener una información más precisa de la cantidad de carbono orgánico, es necesario recurrir a técnicas más avanzadas en el que se utilizan analizadores elementales (Gazulla, Gómez, Rodrigo, y Orduña, 2013).
Además de las propiedades cerámicas intrínsecas de las arcillas, es de suma importancia una adecuada técnica de extracción que evite la contaminación con material no deseado que reduce la calidad de las materias primas. Para un correcto control de ello, es importante un adecuado sistema de explotación, iniciando desde la fase del planeamiento minero, en el cual se debe contemplar todas las peculiaridades del proyecto, actualmente se cuentan con variedad de software que simulan y visualizan los procesos operacionales en base a parámetros propios del área a intervenir (Sepúlveda, Branch, y Jaramillo, 2012).

Debido a la disposición relativamente superficial de los depósitos en la región, lo que incide en una baja relación de descapote, factor que es tenido en cuenta en la elección del sistema de extracción, es común el uso del método de explotación a cielo abierto (Amstrong y Menon, 2001; Herrera, 2006). Una de las características de los mantos explotables es su ubicación geográfica, ya que muchas veces afloran cerca de vías principales de transporte intermunicipal e interdepartamental (Consorcio de estructuración Vial, 2014), lo que ha provocado el interés de empresarios en realizar montajes fabriles, decisión que puede ser compleja dado el crecimiento perimetral de la ciudad, en el que las autoridades territoriales pueden cerrarlas o reubicarlas, a fin de evitar afectaciones futuras a la comunidad (Acuerdo 089 del 2011).

\section{Producción y Costos de la pasta}

\section{cerámica}

La arcilla una vez explotada es llevada a los centros de beneficio. Los procesos cerámicos en el área metropolitana de Cúcuta se dividen en dos procesos: esmaltados y no esmaltados.

La mayor parte de las empresas del gremio cerámico en la región, cerca de 60 de ellas, producen piezas no esmaltadas (Sánchez y Ramírez, 2013), que se caracterizan por el almacenamiento de la materia prima bajo cubierta para evitar la alteración 
por parte de agentes atmosféricos como la lluvia, la radiación solar e incluso el viento. Una vez se cuente con el material se dosifica en el cajón alimentador acorde a las necesidades fabriles, de allí se lleva al molino de martillo para ser molturado y conseguir una granulometría de pasta optima, no obstante, para evitar inconvenientes con sobre-tamaños, se lleva el material hasta un elevador de cangilones donde se vierte sobre un tamiz tipo trommel, el pasante malla 12 es seleccionado y depositado en silos que alimentan el equipo mezclador, el cual dosifica la cantidad de agua agregada a la masa, en función del producto a realizar, posteriormente, la pasta es llevada a la extrusora, la cual cuenta en su boquilla con el molde que proporciona la forma deseada del producto, a continuación las piezas toman un tiempo en secado ya sea natural o artificial, para finalmente pasar a hornos colmena u hornos túneles donde consiguen sinterizar, después de esto, los productos son almacenados, despachados y comercializados.

Además de lo anterior, en el área de estudio se realizan baldosas cerámicas con procesos de esmaltados, en el cual, la arcilla que ingresa a la planta proveniente de la mina, se almacena en cuartos especiales para evitar cualquier alteración, allí se separa acorde a su tipología y posteriormente se lleva a la zona de cargue de acuerdo a las concentraciones indicadas en la formula composicional, seguido se le agrega agua y desfloculante para iniciar la molienda vía húmeda, a fin de proporcionarle fluidez y demás características al compuesto, a esta fase de la mezcla comúnmente se le llama barbotina (Moortgat et al., 1994), después de esto, la masa es enviada a un atomizador donde se le inyecta una corriente de aire caliente, consiguiendo eliminar buena parte del agua, dando como resultado la pasta cerámica. Esta pasta es tamizada para lograr separar las partículas que no cumplen con la granulometría requerida, un proceso similar se realiza con la preparación de los esmaltes (sacmi iberica S.A., 2001). A continuación, la pasta se almacena en silos, que surten los equipos de prensado, los cuales tienen como objetivo la conformación de la baldosa a partir de la pasta atomi- zada, mediante la eliminación del aire presente en etapas anteriores a través de la compactación, mejorando de esta forma su resistencia. Luego del prensado la pieza es secada artificialmente, hasta lograr remover el agua de la mezcla, posteriormente, se aplica el engobe y el esmalte, para esto se suele emplear las técnicas de campana y disco, dependiendo de la tipología del producto.

Para el decorado de las baldosas se adhiere tinta serigráfica a la cara superior de la baldosa esmaltada, ejerciendo una presión mediante un barrido de espátulas sobre la pantalla dejando así impreso el diseño sobre la pieza. Las baldosas son transportadas por bandas hasta un horno de rodillos en el que son cóccidas a altas temperaturas mientras se están moviendo, finalmente el producto acabado es seleccionado y empacado para ser llevado a la venta. La Figura 2 resume a través de un diagrama los procesos llevados a cabo para la fabricación de productos esmaltados y no esmaltados en la región.

De acuerdo con algunos empresarios del sector, el control de costos en materia prima se relaciona directamente con la cantidad de pasta que se utiliza para fabricar un metro cuadrado de producto A manera de ejemplo, la tableta que es uno de los artículos cerámicos no esmaltados más representativos en la región (Sánchez and Ramirez, 2013), inicialmente la arcilla pura tiene un costo puesta en planta de $\$ 16$ pesos por kilogramo, después de ser procesada hasta convertirse en pasta su valor aumenta, en la actualidad tiene un precio medio de $\$ 23$ pesos $/ \mathrm{kg}$, en vista a las dimensiones de los productos, las tabletas gastan 30 kilos para conseguir el metro cuadrado, por lo que el costo total de pasta es de $\$ 690$ pesos $/ \mathrm{m}^{2}$; al comparar estos valores con el costo total promedio general de producción, el cual está en los $\$ 5000$ pesos $/ \mathrm{m}^{2}$ en la región, se puede apreciar que el concepto de pasta cerámica suele ser el 14\% del costo total. De igual forma, las baldosas esmaltadas son medidas en metros cuadrados, en el que según cifras obtenidas de uno de los grandes productores del Departamento, para elaborar un metro cuadrado se necesita en promedio $17,5 \mathrm{~kg}$ de 
pasta, cada kilogramo de masa cuesta alrededor de los $\$ 110$ pesos, al comparar este valor con el costo de $\mathrm{m}^{2}$ de baldosa esmaltada, que a nivel nacional se sitúa en los $\$ 7900$ pesos al año 2017, deja ver que la participación del concepto pasta respecto al cos- to global es de $24,37 \%$, es decir cerca de un cuarto. La Tabla 3 deja ver un comparativo de los costos de pasta cerámica, para la producción de un metro cuadrado de baldosas esmaltadas y tabletas (producto no esmaltado).

Figura 2. Flujograma de los procesos industriales para la elaboración de productos esmaltados y no esmaltados.

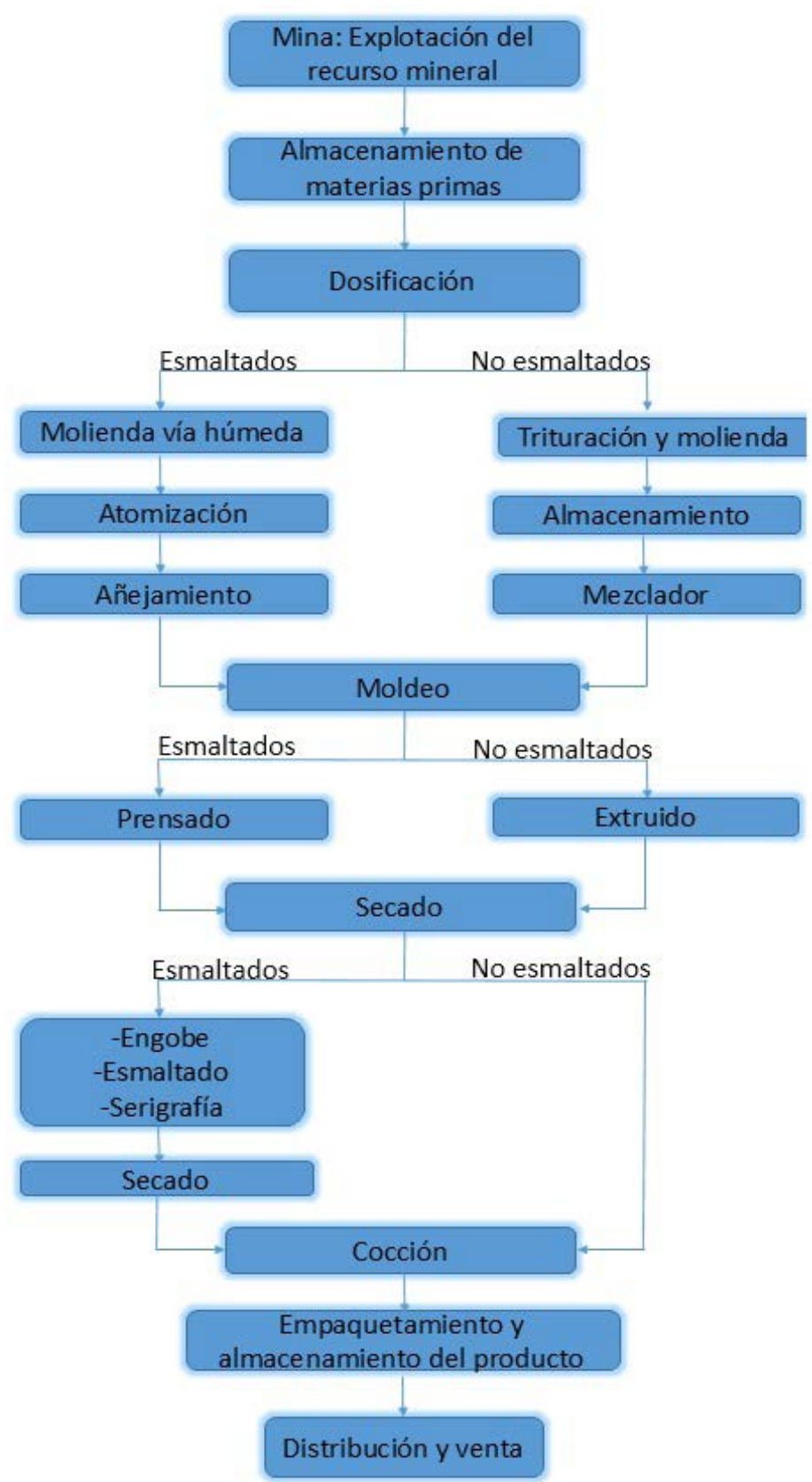


TABLA 3. COMPARATIVO DE COSTOS MATERIA PRIMA ENTRE TABLETA (NO ESMALTADO) Y BALDOSA ESMALTADA. FUENTE: AUTORES.

\begin{tabular}{|c|c|c|c|c|c|}
\hline Producto cerámico & $\begin{array}{c}\text { Costo pasta/ } \\
\text { kg (\$pesos/kg) }\end{array}$ & $\begin{array}{c}\text { Cantidad } \\
\left(\mathbf{k g} / \mathbf{m}^{2}\right)\end{array}$ & $\begin{array}{c}\text { Costo pasta/m2 } \\
\left(\$ \text { pesos } / \mathrm{m}^{2}\right)\end{array}$ & $\begin{array}{l}\text { Costo general } \\
\left(\$ \text { pesos } / \mathbf{m}^{2}\right)\end{array}$ & $\begin{array}{c}\text { Participación } \\
\text { costo (\%) }\end{array}$ \\
\hline Tableta (producto no esmaltado) & 23 & 30 & 690 & 5.000 & 14 \\
\hline Baldosa esmaltada & 110 & 17,5 & 1.925 & 7.900 & 24,37 \\
\hline
\end{tabular}

\section{La economía del sector cerámico en}

\section{el área metropolitana de Cúcuta}

Según Bernal (1991), el desarrollo del sector cerámico involucra el encadenamiento de las actividades de extracción de las materias primas con la manufactureras y por ultimo las ventas, al respecto, el área metropolitana de Cúcuta ha conseguido aglomerar el $91 \%$ de la industria departamental, y en su zona rural se halla el $99 \%$ de la minería de arcilla (Junta metropolitana de Cúcuta, 2015). Estos indicadores, dejan ver la concentración que tiene lugar esta industria en la economía regional, agrupando las unidades productoras de arcillas con las industrias manufactureras, en donde para el año 2012, se contaba con 67 empresas que han desarrollado una gama productos en base a la arcilla, destacándose en tejas, ladrillos, porcelana, material refractario, etc (Sanchez y Ramirez, 2013). Avendaño, Rueda y Paz (2016), señalan este entorno como una fortaleza para el sector fabril que ha servido para impulsar la economía, pero advierte el riesgo de generar afectaciones graves al medio ambiente, que lleven a provocar el rechazo de las comunidades, para evitar lo anterior es recomendable el uso de buenas prácticas empresariales (Sánchez, Gelves, y Romero, 2012).

Una forma de conocer el impacto que genera la Arcilla sobre la economía en la industria regional es siguiendo la metodología aplicada por Mogrovejo et al (2015) y Sánchez (2014) con base a la Encuesta Anual Manufacturera (EAM) dada a conocer por el Departamento Administrativo Nacional de Estadísticas (DANE). Con esta información se analizan varios factores que inciden en la economía de la región, para este estudio se tomaron en cuenta cuatro parámetros principales: el numero establecimientos que se instalaron en la ciudad, la generación de empleo que la industria llegó a crear, la suma de los sueldos y salarios del personal ocupado y finalmente el valor de la producción bruta que tiene gran relación con la generación de riquezas. El presente artículo utilizó la EAM del año 2015 (DANE, 2015), la cual tomó en cuenta la Clasificación Internacional Industrial Uniforme (CIIU) que periódicamente divulga la oficina de estadísticas de la Organización de las Naciones Unidas, para el año 2015 se consideró la revisión 4 de la misma, allí se clasifican las industrias mediante grupos, en la que cada uno posee un código que lo identifica (Marconi, 2006), los productos fabricados a base de minerales no metálicos (arcillas, calizas y demás minerales del gremio cerámico; especialmente las arcillas que es el material de mayor consumo) fueron agrupados cómo el grupo industrial 239 (DANE, 2015), a continuación se analiza dicha información conforme a lo expuesto en la Tabla 4.

La tabla muestra la proporción de la industria cerámica respecto al total del área metropolitana de Cúcuta, se hace evidente que la participación de este sector es mucho mayor frente al segundo más grande de los industriales (Calzado), en la categoría de establecimientos, la industria cerámica posee el 19,2\% de empresas instaladas en la región, cuenta con el $38,1 \%$ del personal ocupado por la industria, su importancia aumenta al concentrar el $40 \%$ de los sueldos y salarios devengados y finalmente la producción bruta es la mayor de los grupos estudiados siendo el $40,6 \%$ del total industrial del área metropolitana. 
TABLA 4. INDICADORES DEL GRUPO 239 EN LA ECONOMÍA INDUSTRIAL DEL ÁREA METROPOLITANA DE CÚCUTA. FUENTE: ENCUESTA ANUAL MANUFACTURERA DEL DANE 2015.

\begin{tabular}{c|c|c|c|c} 
Grupo Industrial & $\begin{array}{c}\text { No. De } \\
\text { establecimientos(a) }\end{array}$ & $\begin{array}{c}\text { Personal } \\
\text { ocupado }^{(\mathbf{b})}\end{array}$ & $\begin{array}{c}\text { Sueldo y salarios en } \\
\text { miles de pesos }^{(\mathrm{c})}\end{array}$ & $\begin{array}{c}\text { Producción bruta en } \\
\text { miles de pesos }^{(\mathrm{d})} \text {. }\end{array}$ \\
\hline Calzado & 14 & 552 & 4730,53 & 45215,69 \\
\hline Minerales no metálicos & 23 & 1.737 & 18348,73 & 344755,07 \\
\hline Total (grupos industriales) & 120 & 4.564 & 45837,99 & 861719,26 \\
\hline
\end{tabular}

Nota: Solo se incluye los dos grupos industriales más grandes del área metropolitana de Cúcuta, a modo de comparación.

(a) Conformados por 10 o más personas ocupadas o con una producción igual o superior a 150,7 millones de pesos de 2015.

(b) Abarca a propietarios, socios familiares, personal permanente, temporal contratado directamente por el establecimiento y a través de empresas especializadas en el suministro de personal y aprendices en etapa práctica.

(c) De los empleados permanentes y temporales medidos.

(d) Sin incluir los impuestos indirectos.

\section{Conclusiones}

La caracterización de los diferentes agentes y/o factores que se involucran en el sector cerámico en el área metropolitana de Cúcuta, se llegó a las siguientes conclusiones:

La ausencia de afloramientos de las formaciones geológicas León o Guayabo, según se observó en la información geológica de la región, encontrándose en su lugar depósitos aluviales y coluviales de tipo cuaternario, explica probablemente la falta de explotaciones de arcilla en el municipio de Puerto de Santander, situación que se puede corroborar en la base de datos de catastro minero, donde no hay registro de títulos o solicitudes de área para el aprovechamiento del mineral, este hecho refleja una carencia en la disposición próxima de materias primas para un óptimo desarrollo de la industria del gres, contrario a lo que sucede en los demás municipio.

Las arcillas presentes dentro del grupo guayabo en los seis sitios analizados mostraron un comportamiento físico cerámico heterogéneo, en el que se evidencia materiales con mayor contenido de desgrasantes que otros, al igual que variación en el porcentaje de contracción en cocido; el uso industrial según la clasificación por absorción de agua, divide las arcillas en dos grupos, en donde se emplearían en procesos diferentes de no ensayarse mezclas que le permitan alcanzar una estandarización de la pasta. Por lo tanto aun cuando la materia prima arcillosa provenga del mismo grupo o formación geológica es apropiado caracterizar de forma independiente, evitando la generalización, ya que esto no da certeza acerca de sus propiedades tecnológicas.

Los porcentajes de participación del grupo cerámico respecto al total industrial en los indicadores económicos de la región, revelan un gran de impacto en la población, lo que lo hace merecedor de ser un agente de vital importancia para jalonar el progreso. No obstante los procesos desarrollados no revelan mayor adelanto tecnológico, por lo que se continua trabajando bajo conductas convencionales, esto se debe probablemente a los bajos costos que genera la materia prima, especialmente en los productos no esmaltados, no estimula la necesidad de innovar, sumado al hecho de contar con parámetros cerámicos con altos estándares de calidad. Sin embargo, dado al panorama actual del mercado globalizado, donde los mejores productos asociados con procesos eficientes frecuentemente debido al uso de modernas tecnologías logran ser competitivos incluso en zonas apartadas, podría afectar la productividad del gremio, por tal razón es importante que el sector disponga de especial importancia al liderazgo en la producción de artículos cerámicos. 


\section{Referencias}

Acuerdo 089 del 2011 (Emitido por el concejo municipal de San Jose de Cúcuta el 30 de diciembre del 2011, de conformidad con la Ley 388 de 1997 y los Decretos 4002 de 2004 y 3600 de 2007) Cúcuta: Concejo municipal de San José de Cúcuta.

Agencia Nacional de Hidrocarburos (ANH) (2010). Open Round Colombia 2010: Catatumbo, 30(1), Bogotá Colombia, p. 2. [Online] Disponible en: http://ronda2010.anh.gov.co/.

Agencia Nacional Minera (2017). Consultas: Agencia Nacional Minera. [Online] Disponible en: https://tramites.anm.gov.co/Portal/pages/consultaListados/ anonimoListados.jsf. [consultado 30 de mayo 2017]

Alvarez Rozo, D. y Sánchez Molina, J. (2014). Desarrollo de revestimientos cerámicos de gres a partir de materias primas de norte de Santander. Cúcuta, Colombia. Facultad de ciencias básicas, UFPS, pp 105-115

Amstrong, J. R. y Menon, R. (2001). Minas y canteras, en Enciclopedia de la Organización Internacional del Trabajo, traducido del inglés por Ministerio de Empleo y Seguridad Social de España, tercera edición, pp. 74.1-74.63. [Online] Disponible en: http://www. insht.es [Consultado 4 de septiembre del 2017].

Avendaño, R., Rueda, G. and Montes, L. (2016). La gestión ambiental en las pymes del sector arcilla en Cúcuta y su área metropolitana, Finanzas y Politica Economica, 8(1) enero-junio, pp. 123-155.

Barrero, D. et al. (2007). Colombian Sedimentary Basins: Nomenclature, boundaries and Petroleum Geology, a New Proposal [e-book], Bogotá: Agencia Nacional de Hidrocarburos A.N.H. [Online] Disponible en: http://www.anh.gov.co/Informacion-Geologica-yGeofisica/Cuencas-sedimentarias/Documents/colombian_sedimentary_basins.pdf [Consultado 20 de junio de 2017]

Bernal, I. (1991). Industria cerámica clásica [e-book]. Bogotá: Servicio nacional de aprendizaje. [Online] Disponible en: http://biblioteca.sena.edu.co/exlibris/ aleph/u21_1/alephe/www_f_spa/icon/40411/ pdf/ceramica_clasica.pdf [Consultado 18 de junio de 2017].

Bracho-Pérez, E. y Escobar-Navarro, M. (2011). Geoquímica de crudos Cretáceos del Lago de Maracaibo, En: XXI Congreso nacional de geoquímica (INAGEQ 2011) Monclova, México, Universidad Autónoma de
Coahuila e Instituo Nacional de Geoquimica, A.C, pp 28 - 37. Disponible en: https://s3.amazonaws. com/academia.edu.documents/30165010/actas_inageq_vol17_2011.pdf?AWSAccessKeyId= AKIAIWOWYYGZ2Y53UL3 A\&Expires=1524850451\&Signature=apuWKpuEFaJyrgguJiIVDwc\%2 Fwec\%3D\&response-content-disposition=inline \%3B\%20filename\%3DGEOQUIMICA_ORGANICA_ APLICADA_A_MADERA_FO.pdf\#page $=39$ [Consultado 20 de junio de 2017].

Cámara de comercio de Cúcuta (2012a). Documento Del Clúster de Cerámica de Norte de Santander. [pdf] Cúcuta, Cámara de comercio de Cúcuta. [Online] Disponible en: http://www.datacucuta.com/PDF/ publicaciones-externas/CLUSTER/ARCILLAS\%20 COMPETITIVAS/DOCUMENTO\%20DEL\%20CLUSTER.pdf [Consultado 12 de agosto de 2017].

Cámara de comercio de Cúcuta (2012b). Documento líneas de acción. [pdf] Cúcuta, Cámara de comercio de Cúcuta. [Online] Disponible en: http://www.datacucuta.com/PDF/publicaciones-externas/CLUSTER/ARCILLAS COMPETITIVAS/DOCUMENTO DEL CLUSTER.pdf [Consultado 4 de septiembre de 2017].

Campos, J. et al (2006). Ahorro de Energía En La Industria Cerámica, Unidad de Planeación Minero Energética e Instituto Colombiano para el Desarrollo de la Ciencia y la Tecnología, pp. 1-28. [Online] Disponible en: http://www.si3ea.gov.co/Portals/0/Gie/Procesos/ ceramica.pdf [Consultado 20 de agosto de 2017].

Catastro Minero Colombiano (2017). Consulta de expediente: Catastro Minero Colombiano. [Online] Disponible en: http://www.cmc.gov.co:8080/CmcFrontEnd/consulta/index.cmc [Consultado 30 de mayo 2017].

Cely, L. y Bolivar, R. (2015). Materia prima para la industria cerámica de Norte de Santander. II. Evaluación del comportamiento térmico y su incidencia en las propiedades tecnológicas, Respuestas, 20(1). [Online] Disponible en: http://respuestas.ufps.edu.co/ ojs/index.php/respuestas/article/view/260 [Consultado el 15 de junio del 2017].

Centro Regional de Estudios Económicos CREE (2005). Notas económicas regionales: Región centro oriente, Bucaramanga Colombia, Banco de la República, pp. 1-10. [Online] Disponible en: <http://www.banrep. gov.co/docum/Lectura_finanzas/pdf/rco_4.pdf>. 
Congreso de la Republica de Colombia (2001) Ley 685 de $20011 / 109$.

Consorcio de estructuración Vial. (2014). Informe geológico-geotécnico: corredor 4 a: Zulia-Cúcuta - Puerto Santander, Agencia Nacional de Infraestructura, p 69. [Online] Disponible en: <ftp://ftp.ani.gov.co/Tercera\%200la/Oca\%C3\%B1a\%20Cucuta/E/EII/EII1/ EII12/EII1213/Geog.\%20y\%20Geot/150204\%20 Geolog\%C3\%ADa\%20Corredor\%204A\%20V\%20 1\%20Zulia-cucuta-pto\%20sant.pdf>.

Consorcio GSG (2015). Plancha 77- Campo Dos, Bogotá: Servicio Geológico Colombiano, SGC. [Online] Disponible en: <http://srvags.sgc.gov.co/Flexviewer/ Estado_Cartografia_Geologica/>.

Consorcio GSG (2015). Plancha 78-Puerto Santander, Bogotá, Servicio Geológico Colombiano. [Online] Disponible en: <http://srvags.sgc.gov.co/Flexviewer/ Estado_Cartografia_Geologica/>.

Corporación Autónoma Regional de la Frontera Nororiental (2011). Capítulo 1. Marco General, en Plan de Acción Ajustado 2007-2011, [e-book]. Cúcuta, Colombia: COORPONOR, pp. 3-95. [Online] Disponible en: $<$ http://corponor.gov.co $>$.

Departamento Administrativo Nacional de Estadística DANE (2015). Clasificación industrial internacional uniforme de todas las actividades económicas, Revisión 4 adaptada para Colombia CIIU Rev. 4 A.C. Bogotá, DANE, pp. 1-497. [Online] Disponible en: <https://www.dane.gov.co/files/nomenclaturas/ CIIU_Rev4ac.pdf>.

Departamento Administrativo Nacional de Estadística DANE (2015). Encuesta Anual Manufacturera 2015, Bogotá, DANE. [Online] Disponible en: <https:// www.dane.gov.co/index.php/estadisticas-portema/industria/encuesta-anual-manufactureraenam>.

Departamento Nacional de Planeación DNP (2007). Agenda interna para la productividad y la competitividad, Norte de Santander, Bogotá, DNP, p 52. [Online] Disponible en: <http://www.mincit.gov.co/ loader.php?IServicio=Documentos\&lFuncion=verP df\&id=23225\&name=Insumos NortedeSantander. pdf\&prefijo=file $>$.

Diaz, E. (2012). Plan de Desarrollo para Norte de Santander "Un Norte Pa'lante" 2012-2015, Cúcuta, Gobernación del norte de Santander, p 176. [Online] Disponible en: <http://www.datacucuta.com/PDF/
publicaciones-externas/PLANESDEDLLO/Un\%20 norte\%20pa\%20lante\%202012-2015.pdf>.

Freddy, J. and Diaz, G. (2011). Efecto de las calizas agregadas a las pastas empleadas en la fabricación de productos cerámicos de construcción del área metropolitana de Cúcuta, Respuestas, 1(1), pp. 38-44. [Online] Disponible a través de fundación Dialnet en <https://dialnet.unirioja.es/servlet/ articulo?codigo $=5364525>$ [Consultado 23 de junio del 2017].

Gazulla, M. F. et al. (2013). Determinación de carbono orgánico en materias primas cerámicas en presencia de siderita, FeCO3, Boleten de la Sociedad Española de Cerámica y Vidrio, 52(3), pp. 5-11. [Online] Disponible en: http://repositori.uji.es/xmlui/bitstream/ handle/10234/83456/59489.pdf?sequence=1 [Consultado 23 de octubre del 2017].

Gelves, J.F., Monroy, R., Sanchez, J. y Ramirez, R.P. (2013). Estudio comparativo de las técnicas de extrusión y prensado como procesos de conformado de productos cerámicos de construcción en el Área Metropolitana de Cúcuta, Boletín de la Sociedad Española de Cerámica y Vidrio, 52(3), pp. 48-54. [Online] <doi: 10.3989/cyv.62013>.

Gelves, J. F., Peña, G., y Sánchez, J. (2009). Comportamiento de las arcillas del Área Metropolitana de Cúcuta sometidas a proceso de moldeo por extrusión, Respuestas, 14(2), pp. 32-38. [Online] Disponible en: http://revistas.ufps.edu.co/ojs/index.php/respuestas/article/view/543 [Consultado 23 de octubre del 2017].

Generalitat Valenciana e Instituto Valenciano de la Edificación (2011). Guía de la baldosa cerámica [e-book], Valencia-España: Generalitat Valenciana, p 108. Disponible en: <http://www.five.es/publicaciones/ GBC/arc/assets/basic-html/index.html\#page4>.

Herrera, J. (2006). Métodos de minería a cielo abierto [pdf], Universidad Politécnica de Madrid. [Online] Disponible en: http://oa.upm.es/10675/1/20111122_ METODOS_MINERIA_A_CIELO_ABIERTO.pdf [Consultado 4 de octubre del 2017].

Instituto Colombiano de Geología y Minería (1998). Memoria del cuadrángulo G-13 Cúcuta, Bogotá, Servicio Geológico Colombiano. [Online] Disponible en: http://srvags.sgc.gov.co/Flexviewer/Estado_Cartografia_Geologica [Consultado 12 de julio del 2017]. 
Instituto Colombiano de Geología y Minería (2011). Geología De Las Planchas 98 - Durania Y 99 - Villa Del Rosario, Norte De Santander - Colombia, Bogotá, Servicio Geológico Colombiano. [Online] Disponible en: http://srvags.sgc.gov.co/Flexviewer/Estado_Cartografia_Geologica/ [Consultado 22 de junio del 2017].

Instituto Colombiano de Normas Técnicas y Certificación (2015). NTC 919, Baldosas Cerámicas: Definiciones, Clasificación, Características y Rotulado, Bogotá, INCONTEC, $56 \mathrm{p}$.

Jiménez, C. (2008). La frontera colombo-venezolana: una sola región en una encrucijada entre dos estados. Reflexión Política, 10, pp. 258-272. [Online] Disponible a través de: Red de Revistas Científicas de América Latina, el Caribe, España y Portugal <http://www.redalyc.org/pdf/110/11002019. pdf $>$ [Consultado 13 de septiembre del 2017].

Junta metropolitana de Cúcuta (2015). Área viva, Región sostenible, Cúcuta, Área metropolitana de Cúcuta, AMC. [Online] Disponible en: http://www.amc.gov. co/areametro/acuerdos/archivo/0757703.pdf.

Lores, M. T. et al. (1997). Estudio de la eliminación de materia orgánica en arcilla mediante tratamiento térmico. Análisis del proceso a diferentes escalas. Boletín de la Sociedad Española de Cerámica y Vidrio, 36(4), pp. 419-424. [Online] Disponible en: http:// boletines.secv.es/upload/199736419.pdf [Consultado 23 de octubre del 2017].

Marconi, S. (2006). Clasificación Industrial Internacional Uniforme de todas las actividades económicas (CIIU), Revisión 4 Estructura y notas explicativas. En: CELADE y la DGEEC de Paraguay, Seminario regional de intercambio de experiencias en materia de clasificadores y nomenclatura, Santiago de Chile, 13 al 14 de diciembre 2010.

Milošević, M., Logar, M., Kaluđerović, L., Jelić I. (2017). Characterization of clays from Slatina (Ub, Serbia) for potential uses in the ceramic industry. Energy procedia, 125 (2017), pp 650-655. Doi: https://doi. org/10.1016/j.egypro.2017.08.270 [Consultado 30 de agosto del 2017].

Mogrovejo, J., Bastos, L. y Pabón, J. (2015). Impacto económico del sector cerámico en San José de Cúcuta, Universidad \& Empresa [e-journal], 17(29), pp 157180. Doi: dx.doi.org/10.12804/rev.univ.empresa.29.2015.07 [Consultado 20 de junio del 2017].
Moortgat, G., Vancaster, N., Vandeneede, V., y Cambier, F. (1994). La caracterización de barbotinas arcillosas utilizando medidas de resistencia a la polarización. En III congreso mundial de la calidad del azulejo y del pavimento cerámico (pp. 211-221). Castellón, España. [Online] Disponible en: <http://www.qualicer.org/recopilatorio/ponencias/pdfs/9423151s. pdf>.

Ochoa, A. et al. (2016). Geología de la plancha 87 - Sardinata departamento de Norte de Santander, Bogotá, Servicio Geológico Colombiano SGC, 196 p.

Ortega-lara, A. et al. (2012). Peces de la cuenca del río Catatumbo, cuenca del Lago de Maracaibo, Colombia y Venezuela, Biota Colombiana, 13(1), pp. 71-98. [Online] Disponible a través de: Red de Revistas Científicas de América Latina, el Caribe, España y Portugal <http://www.redalyc.org/articulo. oa?id=49125118003> [consultado 9 de junio del 2017].

Oviedo, J. et al. (2016). Geología de la plancha 88 - Cúcuta a escala 1:100.000 departamento de Norte de Santander - Colombia, Bogotá: Servicio Geológico Colombiano, $156 \mathrm{p}$.

Perdomo, J., Malaver, F. y Stalin, J. (2002). Proyecto equipo negociador "ALCA" en su clústers estratégicos regionales aplicación al clúster calzado para dama - Cúcuta, Bogotá, Centro de Investigaciones para el desarrollo, UNAL. [Online] Disponible en: http://www.cid.unal.edu.co/files/publications / CID200211pepreq_a.pdf [consultado 4 de agosto del 2017].

Rivas, Z. et al. (2009). Nitrógeno y fósforo totales de los ríos tributarios al sistema lago de Maracaibo, Venezuela, Interciencia, 34(5), pp. 308-314. [Online] Disponible a través de: Red de Revistas Científicas de América Latina, el Caribe, España y Portugal <http:// www.redalyc.org/articulo.oa?id=33911403002> [Consultado 20 de junio del 2017].

Rozo Rincón, S. M., Sánchez Molina, J. y Gelves Díaz, J. F. (2015). Evaluación de minerales alumino silicatos de Norte de Santander para fabricar piezas cerámicas de gran formato, Facultad de Ingeniería, 24(38), pp. 53-61. Disponible a través de scielo: <http://www.scielo.org.co/scielo.php?script=sci_ arttext\&pid=S0121-11292015000100005\&lng=en $\& n r m=i s o \&$ tlng=es $>$ [Consultado 16 de octubre del 2017]. 
Saavedra, J. S. (2016). Modelamiento geológico minero y cálculo de reservas de la mina támesis $i$ en las veredas de agualinda y juan frio, de los municipio de los patios y villa del rosario, norte de santander. Tesis pregrado Geologia. Universidad de Pamplona.

Sacmi Iberica S.A. (2001). Tecnología cerámica aplicada. Vol I, Faenza Editrice Ibérica S.L. Faenza RA, Italia: Litográfica Faenza S.R.L.

Sánchez, A. (2014). Crisis en la frontera, Cartagena Colombia, Banco de la Republica CEER-Cartagena, 197. [Online] Disponible en: <http://www.banrep.gov. co/docum/Lectura_finanzas/pdf/dtser_197.pdf>.

Sánchez, E. et al. (1990). Criterios de selección de materias primas para la fabricación de pavimentos y revestimientos cerámicos, Cerámica Información, No. 157, julio-agosto, pp. 13-21.

Sánchez, J. (2017). Cisco de café como posible material sustituto de arcilla en la fabricación de materiales cerámicos de construcción en el área metropolitana de Cúcuta IV. En: UFPS (Universidad Francisco de Paula Santander), Semana Internacional y XII Semana de Ciencia, Tecnología e innovación, Cúcuta, Colombia.

Sánchez, J. y Díaz, J. (2011). Introducción A Los Hornos Utilizados En La Industria Cerámica Tradicional, Cúcuta, Colombia: Universidad Francisco de Paula Santander.

Sánchez, J., Gelves, J. F. y Ramirez, R. P. (2012). Implementación de un sistema tipo SCADA para mejorar los procesos de secado y cocción de la ladrillera SIGMA LTDA, Revista Colombiana de Tecnologías de Avanzada, 2(20), pp. 80-85. [Online] Disponible en: http:// revistas.unipamplona.edu.co/ojs_viceinves/index. php/RCTA/article/view/192 [Consultado 26 de octubre del 2017].

Sánchez, J. y Ramirez, R. (2013). El Clúster De La Cerámica Del Área Metropolitana De Cúcuta. Cúcuta, Colombia: Universidad Francisco de Paula Santander.

Sánchez Molina, J., Alvarez Rozo, D. y Sarabia-Guarin, A. (2016). Evaluación de materias primas utilizadas en la fabricación de baldosas de gres en el sector cerámico de Norte de Santander (Colombia), Respuestas, 21(2), pp. 48-56. [Online] Disponible en: http:// dx.doi.org/10.22463/0122820X.776

Sánchez Molina, J., Gelves Díaz, J. F. y Romero Arcos, Y. A. (2012). Caracterización tecnológica y del talento humano de las empresas fabricantes de cerámica roja ubicadas en el área metropolitana de Cúcuta, Respuestas, 17(2), pp. 71-80. [Online] Disponible en: <http://revistas.ufps.edu.co/ojs/index.php/ respuestas/article/viewFile/423/448>.

Sarabia Guarin, A., Sánchez Molina, J. y Leyva Díaz, J. C. (2017). Uso de nutrientes tecnológicos como materia prima en la fabricación de materiales de construcción en el paradigma de la economía circular, Respuestas, 22(1), pp. 6-16. [Online] <https://doi. org/10.22463/0122820X.815>.

Sepúlveda, G. F., Branch, J. and Jaramillo, P. (2012). Planning of open pit mines through stochastic optimization, Boletín de Ciencias de la Tierra, pp. 107-113. [Online] Disponible a través de scielo en: http:// www.scielo.org.co/scielo.php?script=sci_arttext $\&$ p $\mathrm{id}=\mathrm{S} 0120-36302012000100008$ [Consultado 4 de septiembre del 2017].

Servicio Geológico Colombiano (2017a). Geología de las planchas 87 (Sardinata) y 88 (Cúcuta), en: UFPS (Universidad Francisco de Paula Santander), I seminario de las Geociencias y la Metrología, Subred de geociencias y minería - RCM. Cúcuta, Colombia, p. 54.

Servicio Geológico Colombiano (2017b). Mapa geológico colombiano. SGC. [On line] Disponible en: http:// srvags.sgc.gov.co/Flexviewer/Mapa_Geologico_Colombia/ [Consultado 16 de junio del 2017].

Sistema de Información Minero (2017). Mapa Ingeominas, Bogotá. [Online] Disponible en: <http://www. simco.gov.co/Inicio/CatastroMineroColombiano/ MapaIngeominas/tabid/376/Default.aspx> [Consultado 15 de junio de 2017].

SIMEC (2012). Entregable 2: Arcillas competitivas, Norte de Santander [pdf], Cúcuta. [Online] Disponible en: $<$ http://www.si3ea.gov.co/Portals/0/Gie/Procesos/ceramica.pdf> [Consultado 4 de septiembre del 2017].

Urdanet, A. y Maracaibo, L. (1988). San José de Cúcuta en el comercio marabino del siglo XIX, Boletín Americanista, No 38, pp. 247-258. [Online] Disponible en: http://revistes.ub.edu/index.php/BoletinAmericanista/article/view/12754/15721 [Consultado 4 de agosto del 2017].

Uribe, A. (2002). Decreto 2390, Bogotá: Ministerio de minas y energía. [Online] Disponible en: https://www. anm.gov.co/.

Valera, A. (2016). Etnohistoria de la Cuenca del Lago De Maracaibo [e-book]. Cabimas, Venezuela: Univer- 
sidad Nacional Experimental Rafael Maria Baralt. [Online] Disponible en: <http://150.185.9.18/fondo_editorial/images/PDF/RafaelMariaBaralt/Etnohistoria del la Cuenca del Lago de Mcbo. - Argenis Valera 1.pdf> [Consultado 20 de junio del 2017].

Vallejo, L. et al. (2002). Ingeniería Geológica. Madrid, España: Pearson Educación, S.A.

Villamizar, W. (2016). Plan de Desarrollo para Norte de Santander 2016-2019 "Un Norte Productivo Para Todos", Cúcuta: Gobernación del Norte de Santander. [Online] Disponible en: <http://www.sisubregionalns. gov.co/files/sid_Desarrollo_territorial/Plan_de_desarrollo_para_Norte_de_Santander_2016-2019. pdf> [Consultado 23 de junio del 2017].

\section{PARA CITAR ESTE ARTÍCULO TO REFERENCE THIS ARTICLE / PARA CITAR ESTE ARTIGO /}

Flórez-Vargas, A.O.; Sánchez-Molina, J.; Blanco-Meneses, D.S. (2018). Las arcillas de las formaciones geológicas de un área metropolita, su uso en la industria cerámica e impacto en la economía regional. Revista EIA, 15(30), julio-diciembre, pp. 133-150. [Online]. Disponible en: https://doi.org/10.24050/ reia.v15i30.1219 